\title{
Praziquantel Associado a Alcaloide no Tratamento da Esquistossomose: prospecção tecnológica em uma perspectiva inovadora
}

\author{
Praziquantel Associated With Alkaloid in the Treatment of \\ Schistosomosis: technological prospection in na innovative perspective
}

\author{
Pedro da Silva Gerônimo Neto ${ }^{1}$ \\ João Marcelo Saraiva Ferreira ${ }^{1}$ \\ Pablo de Matos Monteiro ${ }^{1}$ \\ Maria da Glória Almeida Bandeira ${ }^{1}$ \\ Jaqueline Silva Nascimento ${ }^{1}$ \\ ${ }^{1}$ Universidade Federal do Maranhão, São Luís, MA, Brasil
}

\begin{abstract}
Resumo
A esquistossomose é uma doença negligenciada desencadeada pelo Schistosoma mansoni. O praziquantel (PZQ) é o fármaco de primeira escolha no tratamento da esquistossomose, além de outras doenças negligenciadas causadas por helmintos. Contudo, a dificuldade de adesão ao tratamento e o uso incorreto desse medicamento têm demonstrado resistência do parasito ao praziquantel. Para a elaboração deste artigo, realizou-se uma prospecção de patentes e de artigos dos principais estudos relacionados ao praziquantel. A busca foi realizada nas bases do Instituto Nacional da Propriedade Industrial (INPI) do Brasil, do European Patent Office (Espacenet), do Derwent Innovations Index, do World Intellectual Property Organization (WIPO) e também em artigos indexados no Scielo e no Scopus. Usouse as mesmas palavras chave para as buscas nas bases de patente e nas bases de artigo científico para o período compreendido entre 2008 a 2018. O número de patentes relacionadas ao praziquantel é considerado baixo quando esses resultados são comparados com o número de artigos publicados. O Brasil apresentou o maior número de artigos publicados na base de dados Scielo para o período em estudo, entretanto, isso não se reflete aos pedidos de patentes, dos quais países, como Estados Unidos e China, são os maiores detentores.
\end{abstract}

Palavras-chave: Praziquantel. Patentes. Artigos Científicos.

\begin{abstract}
Schistosomiasis is a neglected disease triggered by Schistosoma mansoni. Praziquantel (PZQ) is the drug of choice in the treatment of schistosomiasis, in addition to other neglected diseases caused by helminths, however, the difficulty in adhering to the treatment and the incorrect use of this medication has demonstrated the parasite's resistance to praziquantel. For the preparation of this article, patents and articles from the main studies related to praziquantel were prospected. The search was carried out on the basis of the National Institute of Industrial Property (INPI) of Brazil, the European Patent Office (Espacenet), the Derwent Innovations Index, the World Intellectual Property Organization (WIPO) and also on articles indexed in Scielo and Scopus. The same keywords were used for searches in the patent bases and in the scientific article bases for the period from 2008 to 2018. Brazil presented the largest number of articles published in the SCIELO database for the period under study, however this is not reflected in patent applications, in which countries such as the United States and China are the largest holders of patent applications.
\end{abstract}

Keywords: Praziquantel. Patent. Scientific Article.

Área Tecnológica: Prospecção Tecnológicas de Assuntos Específicos. 


\section{Introdução}

As doenças negligenciadas formam um grupo de doenças infecciosas causadas por parasitas, bactérias, vírus ou fungos que afetam mais de um bilhão de pessoas em todo o mundo, sendo endêmicas em 149 países. No Brasil, as Regiões Norte e Nordeste, as mais pobres do país, apresentam alta prevalência dessas doenças (BRASIL, 2018). Além disso, por essas doenças atingirem, principalmente, países em desenvolvimento, os investimentos em pesquisa e tecnologia para o controle, a prevenção e o tratamento medicamentoso são baixos (SANTOS et al., 2017). Entre essas doenças se encontra a esquistossomose, também conhecida como "xistose", "barriga d'água" ou "doença dos caramujos" (SILVA; NICOLETTI, 2013).

A esquistossomose mansoni é uma doença parasitária que possui como agente etiológico o Schistossoma mansoni, apresentando alta incidência nos países em desenvolvimento, de clima tropical e com elevadas temperaturas (SILVA; NICOLETTI, 2013). No mundo, a doença ocorre em 54 países, destacando-se os países da África, do Leste do Mediterrâneo, da América do Sul e do Caribe; no Brasil, apresenta incidência em todo o território, sendo as principais áreas endêmicas os estados do Rio Grande do Norte, da Paraíba, de Pernambuco, de Alagoas, de Sergipe, da Bahia, do Espírito Santo, do Maranhão e de Minas Gerais. A expansão da doença pelo Brasil se deu a partir do litoral devido aos movimentos migratórios (SOCIEDADE BRASILEIRA DE INFECTOLOGIA, 2018; BRASIL, 2019).

Quanto ao modo de transmissão, o indivíduo, pode adquirir a infecção quando entra em contato com água doce contaminada pelo caramujo que contém os vermes causadores dessa doença, sendo que, estes, uma vez dentro do organismo da pessoa, se não tratados, podem evoluir e provocar algumas complicações típicas, como: aumento do fígado, do baço entre outras (BRASIL, 2019).

O tratamento medicamentoso da esquistossomose não é diferente da terapêutica usual, mas sempre foi limitado pela dificuldade em encontrar agentes quimioterápicos com alta eficácia e tolerabilidade. O praziquantel $(\mathrm{PZQ})$ é o fármaco de primeira escolha no tratamento da esquistossomose, além de outras doenças causadas por helmintos, apesar dos efeitos adversos que podem provocar e da grande resistência dos parasitas para com esse medicamento (ANDREWS, 1985; SILVA et al., 2017).

O uso disseminado e incorreto desse fármaco para o tratamento de esquistossomose, associado aos casos frequentes de reinfecção, proporciona preocupações quanto ao crescente desenvolvimento de resistência do parasito às terapias medicamentosas, como já foi observado em certas regiões endêmicas. Dessa forma, existe um certo incentivo pela procura e pela elaboração de novos fármacos anti-helmínticos. Portanto, como alternativa para diminuir o aumento de casos das doenças negligenciadas, é importante o estudo de análise molecular de fármacos já existentes, visando a maiores atividades biológicas para o combate a essas doenças (CORDEIRO; PINHEIRO, 2016).

De fato, as modificações na estrutura química do PZQ abrem um campo de estudo para um melhor tratamento contra esquistossomose, o que gera a possibilidade de serem testadas novas moléculas com potencial anti-helmíntica, provocando, assim, melhor eficácia quanto à ação medicamentosa. Dessa forma, uma possível reação química de associação entre o PZQ e o alcaloide atanina pode potencializar a atividade antiparasitária dos fármacos e diminuir os 
mecanismos de resistência do parasita para com o medicamento, fazendo com que haja maior eficácia na terapêutica, agindo sobre as diversas formas de vida do parasita Schistossoma mansoni.

Quanto à sua farmacocinética, o PZQ é um fármaco pouco solúvel em água, entretanto é facilmente absorvido pelo trato gastrointestinal (ANDREWS, 1985). Porém, altas doses orais são necessárias para superar o metabolismo de primeira passagem e, desse modo, obter suficientes concentrações do fármaco no tecido larval (LEOPOLD et al., 1978). O praziquantel é um fármaco classificado na classe II do SCB, ou seja, possui baixa solubilidade e alta permeabilidade, sendo a taxa de dissolução um fator limitante para sua absorção (SILVA, 2008). Além disso, o PZQ é um fármaco fotossensível, higroscópico e extremamente amargo e, como um dos públicos-alvo do medicamento são as crianças, é importante que o revestimento mascare o sabor, de modo a obter uma maior adesão ao tratamento (MERCK INDEX, 2001; WOELFLE et al., 2011). Dessa forma, nos últimos anos, muitas pesquisas têm sido realizadas com o objetivo de melhorar a solubilidade e as propriedades biofarmacêuticas do praziquantel (SILVA, 2008).

Diante do que foi exposto, há procura por novas formulações envolvendo o PZQ, como sugerido por Fonseca (2012), que afirma a busca do Ministério da Saúde pelo desenvolvimento do produto praziquantel em forma de suspensão, já que, por meios de estudos farmacotécnicos, isso pode se tornar realidade, garantindo maior adesão terapêutica dos pacientes ao tratamento. É importante destacar que durante o desenvolvimento de uma suspensão, deve-se garantir que o fármaco a ser suspenso tenha um tamanho de partícula bastante reduzido com intuito de assegurar uma baixa velocidade de sedimentação (ANSEL; POPOVICH; ALLEN, 2000).

É evidente no Brasil que os estudos de prospecção ainda são pouco explorados, apesar da sua importância no âmbito científico (CARVALHO et al., 2020). Assim, Pereira et al. (2015) enfatizam que as bases de patentes e de artigos científicos são importantes para a aquisição de informações tecnológicas e científicas, respectivamente, podendo ser acessadas em qualquer momento e lugar, evitando a perda dos dados. A produção de novos fármacos e medicamentos requer a prospecção de informações obtidas em artigos científicos e patentes. $\mathrm{O}$ objetivo principal desta prospecção é relatar e demonstrar a produção científica, bem como os depósitos de patentes sobre a modificação molecular em estudo e sobre as formas farmacêuticas do medicamento praziquantel, tendo em vista apresentar a evolução dessas produções no período de 2008 a 2018.

Este estudo baseia-se no desenvolvimento de novos medicamentos como alternativas para o tratamento da esquistossomose. De acordo com os resultados, espera-se que essa modificação molecular seja inovadora, possibilitando uma possível patente e, posteriormente, a difusão dessa tecnologia.

\section{Metodologia}

A presente prospecção tecnológica foi realizada com base nos pedidos de patente depositados no banco de dados brasileiro do Instituto Nacional da Propriedade Industrial (INPI) do Brasil, visando à análise das patentes depositadas em território nacional. Somado a isso, também foram realizadas pesquisas nas bases de dados European Patent Office (Espacenet), sendo conhecida por possuir pedidos de patentes por aproximadamente 100 países e na Derwent Innovations Index, que possui mais de 30 milhões de invenções documentadas. Além disso, foi acessada 
a base de dados internacional World Intellectual Property Organization (WIPO), uma vez que esta possui maior abrangência de patentes.

Para a pesquisa de artigos científicos, foram utilizados os bancos de dados da Scielo, importante biblioteca eletrônica que reúne variados periódicos científicos brasileiros, e da Scopus, que se trata de uma importante base de dados de literatura que abrange diversas áreas do conhecimento.

Neste estudo é apresentado um monitoramento em patentes. A busca foi realizada no mês de maio e junho do ano de 2019, sendo o período analisado de 2008 a 2018, utilizando-se combinações de termos inerentes ao fármaco praziquantel em Título e/ou Resumo e contendo todas as palavras. Sendo assim, como descritores do estudo, foram usados termos associados a esse medicamento, tanto para patentes como para busca de artigo, como "praziquantel"; "praziquantel and schistosomiasis", "praziquantel and antiparasitics" e "praziquantel and anthelmintic", para buscar as patentes e as publicações que relacionam o medicamento com sua atividade farmacológica; "praziquantel and atanine", para buscar patentes e artigos com a associação do praziquantel com a atanina; e "praziquantel and suspension" sobre a formulação do PZQ nessa forma farmacêutica. Para as buscas nas plataformas internacionais, foram usados os termos em língua inglesa e, na plataforma nacional, os termos foram usados na língua portuguesa.

Os periódicos decorrentes da busca foram quantificados e analisados com base na distribuição dos países e pela área de conhecimento. Paralelamente, as patentes encontradas foram quantificadas e, para melhor análise, foram distribuídas em patentes depositadas por países, ano, área de conhecimento e por Classificação Internacional de Patentes (CIP).

A análise de dados se deu inicialmente pela exportação dos resultados obtidos em uma planilha do Microsoft Excel 2016. Posteriormente, os resultados foram organizados em tabelas e em gráficos, utilizando-se o Microsoft Word 2016.

\section{Resultados e Discussão}

A prospecção tecnológica, além de mapear o desenvolvimento científico e tecnológico, procura somar valores às informações atuais para que sejam capazes de influenciar na tomada de decisões de uma indústria ou empresa na elaboração de suas políticas de inovação.

Para o começo da análise de patentes sobre a modificação molecular em estudo e da forma farmacêutica proposta pelo Ministério da Saúde do praziquantel, pode-se observar que, de acordo com a Tabela 1, a base World Intellectual Property Organization (WIPO) é a que detém o maior número de patentes sobre essa temática, com 297 patentes encontradas, posteriormente vem a European Patent Office (Espacenet) com 274 e a Derwent Innovations Index com 228 patentes.

Tabela 1 - Número de patentes por palavras-chave em título e resumo encontradas nas bases de dados utilizadas para a pesquisa no período de 2008 a 2018

$\begin{array}{ccccc}\text { Palavras-chave } & \text { INPI } & \text { EsPaCENET } & \text { Derwent } & \text { WIPO } \\ \text { Praziquantel } & 14 & 234 & 204 & 244 \\ \text { Praziquantel and schistosomiasis } & 0 & 11 & 12 & 9 \\ \text { Praziquantel and anthelmintic } & 0 & 19 & 7 & 30\end{array}$




\begin{tabular}{ccccc} 
PalaVRas-chave & INPI & ESPACENET & DeRWENT & WIPO \\
Praziquantel and antiparasitics & 2 & 0 & 0 & 0 \\
Praziquantel and atanine & 0 & 0 & 0 & 0 \\
Praziquantel and suspension & 1 & 10 & 5 & 14 \\
TOTAL & 17 & 274 & 228 & 297 \\
\hline
\end{tabular}

Fonte: Elaborada pelos autores deste artigo (2019)

No Instituto Nacional da Propriedade Industrial (INPI), foram encontrados 17 registros de patentes. Como o Brasil faz parte do Tratado de Cooperação de Patentes (PCT), é possível que algumas patentes que foram encontradas na WIPO sejam as mesmas que estão no INPI, pois têm o mesmo campo de abrangência devido ao acordo.

Esse fato pode ser explicado, pois, de acordo com Kandil (2004), há a divulgação de que o custo para o desenvolvimento de medicamentos é muito alto, o exagero desses custos serve aos interesses internacionais de duas formas: para justificar os altos preços de seus medicamentos e para desencorajar companhias farmacêuticas de países em desenvolvimento, como o Brasil, de investir na descoberta de novos medicamentos devido ao custo proibitivo.

$\mathrm{Na}$ base de dados WIPO, de acordo com Nascimento et al. (2017), é possível se fazer consultas em 65 milhões de documentos de patentes, sendo que 3,2 milhões publicadas na plataforma são por via do Tratado de Cooperação de Patentes (PCT). Com isso, demonstra-se o porque de nessa plataforma se obter a maior quantidade de resultados encontrados (297).

Ainda de acordo com a Tabela 1, a palavra-chave "Praziquantel" é a que mais se destaca, de forma geral, obtendo maior quantidade na plataforma WIPO com 244 patentes, 234 no Espacenet e 204 no Derwent. Entretanto, no Brasil a produção de patentes nessa temática não é expressiva em relação as outras bases, obtendo apenas 14 patentes encontradas.

Diante disso, conforme demonstrado na Tabela 1, é interessante frisar que, na busca por patentes com o descritor "Praziquantel and atanine" em título e resumo, não foram identificados trabalhos em nenhuma das bases. O que demonstra que a possível associação molecular, com a realização dos devidos testes, pode caracterizar uma ideia inovadora. E a partir dessa ideia, podem surgir novos trabalhos a fim de sintetizar e de testar a molécula em estudo.

A Figura 1 apresenta a distribuição dos pedidos, de acordo com os anos de depósito na plataforma INPI, compreendido entre o período de 2008 a 2018. Os depósitos de patentes na base de dados brasileira começaram com um documento em 2008, até chegar a quatro documentos. Nesse intervalo de tempo, percebe-se que houve uma variação do número de patentes referentes ao termo "Praziquantel", porém, em 2018, não há registros de patente.

As patentes depositadas em 2017 demonstram a utilização da combinação do praziquantel com outros fármacos como o timolol, imidacloprido e moxidectina. Porém, nenhuma dessas relatam alterações moleculares, mas apenas a criação de formulações com diferentes concentrações dos fármacos, o que é possível inferir que patentes sobre estudos químicos farmacológicos envolvendo o PZQ são inexistentes no Brasil. 
Figura 1 - Distribuição dos pedidos de patentes de acordo com os anos de depósito na base de dados INPI utilizando o termo "Praziquantel"

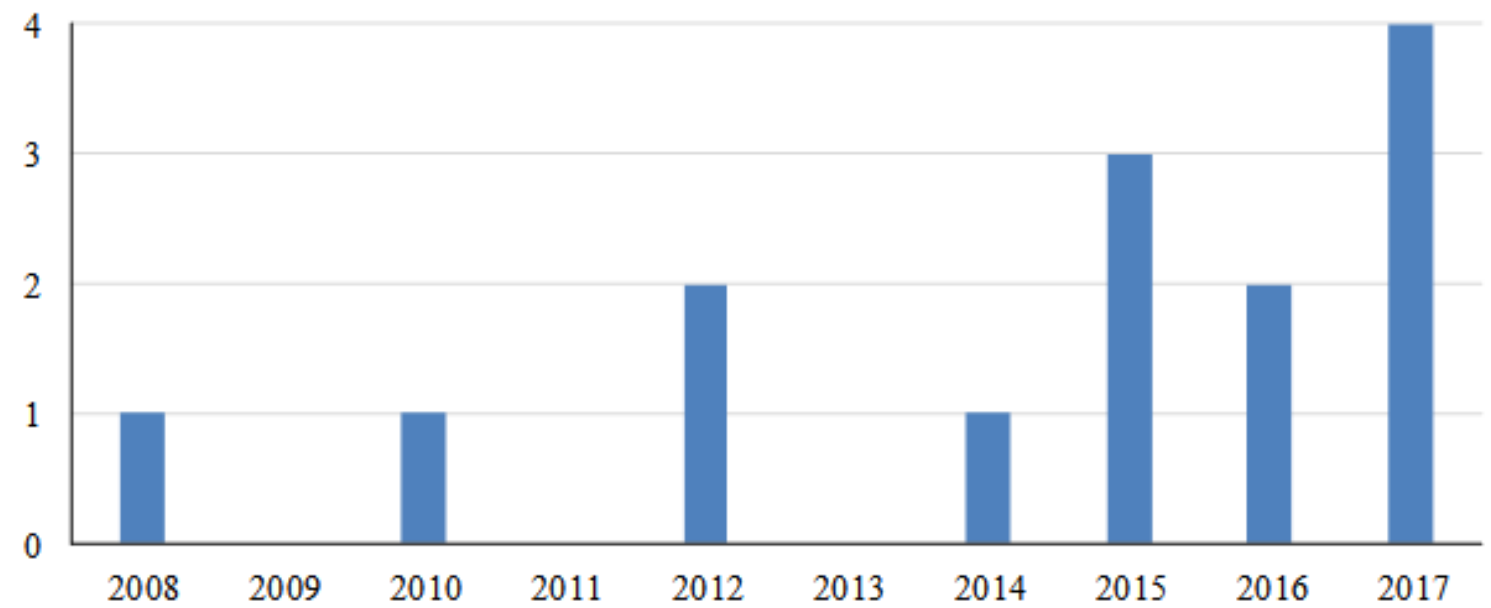

Fonte: Elaborada pelos autores deste artigo (2019)

A publicação de 2008 é a única patente encontrada na base INPI utilizando o termo "Praziquantel and suspension”. Esse documento descreve em seu resumo sobre a invenção de uma suspensão oriunda da combinação de praziquantel e sulfóxido de albendazol, com aplicação por via oral ou intrarruminal para animais de criação, visando ao tratamento ou à prevenção de infecções por cestoide. Observa-se que, desde 2008, há pesquisas envolvendo a criação de novas formas farmacêuticas para o tratamento com o PZQ, seja para uso veterinário ou em humanos.

A Tabela 2 retrata a área de conhecimento para 204 patentes utilizando a palavra-chave "Praziquantel" na plataforma Derwent, em que foram classificadas em 11 áreas de conhecimento. Observa-se que 154 documentos, 99\%, encontram-se na área de "Chemistry", "Pharmacology Phamacy" (141 registros) com 90\%, e a "Agriculture" apresentou 67 documentos gerando um percentual de $43 \%$.

Tabela 2 - Áreas de conhecimento baseado na contagem de registro de patentes na plataforma DERWENT com a palavra-chave "Praziquantel" para o período compreendido de 2008 a 2018

\begin{tabular}{ccc} 
ÁREAS DE ConHecimento & Contagem do Registro & $\mathbf{\%}$ DE $\mathbf{2 0 4}$ \\
Chemistry & 154 & $99 \%$ \\
Pharmacology Pharmacy & 141 & $90 \%$ \\
Agriculture & 67 & $43 \%$ \\
Polymer Science & 48 & $31 \%$ \\
Biotechnology applied Microbiology & 16 & $10 \%$ \\
Engineering & 11 & $7 \%$ \\
Food Science Technology & 11 & $7 \%$ \\
Instruments Instrumentation & 10 & $6 \%$ \\
Computer Science & 2 & $1 \%$ \\
Materials Science & 1 & $0,6 \%$ \\
Water Resources & 1 & $0,6 \%$ \\
\hline
\end{tabular}

Fonte: Adaptada de Derwent Innovations Index (2019) 
A Classificação Internacional de Patentes (CIP) é uma ferramenta que, além de uniformizar as variadas temáticas no âmbito tecnológico para uma linguagem acessível, serve como uma fonte de recuperação de patentes pelos usuários (JANNUZZI; SOUZA, 2008).

Os resultados encontrados na plataforma Derwent estão, principalmente, na subclasse A61K (preparações para finalidades médicas, odontológicas ou higiênicas) e na subclasse A61P (atividade terapêutica específica de compostos químicos ou preparações medicinais). Já as patentes depositadas no INPI estão, principalmente, na subclasse A61K (preparações para finalidades médicas, odontológicas ou higiênicas) e na subclasse C07D (preparação de compostos macromoleculares).

A Figura 2 avalia a quantidade de patentes depositadas na base da plataforma WIPO com relação aos países que desenvolvem inovação tecnológica para a temática em estudo.

Figura 2 - Número de documentos de patentes recuperados na base WIPO com a palavra-chave "Praziquantel" por países, para o período compreendido de 2008 a 2018

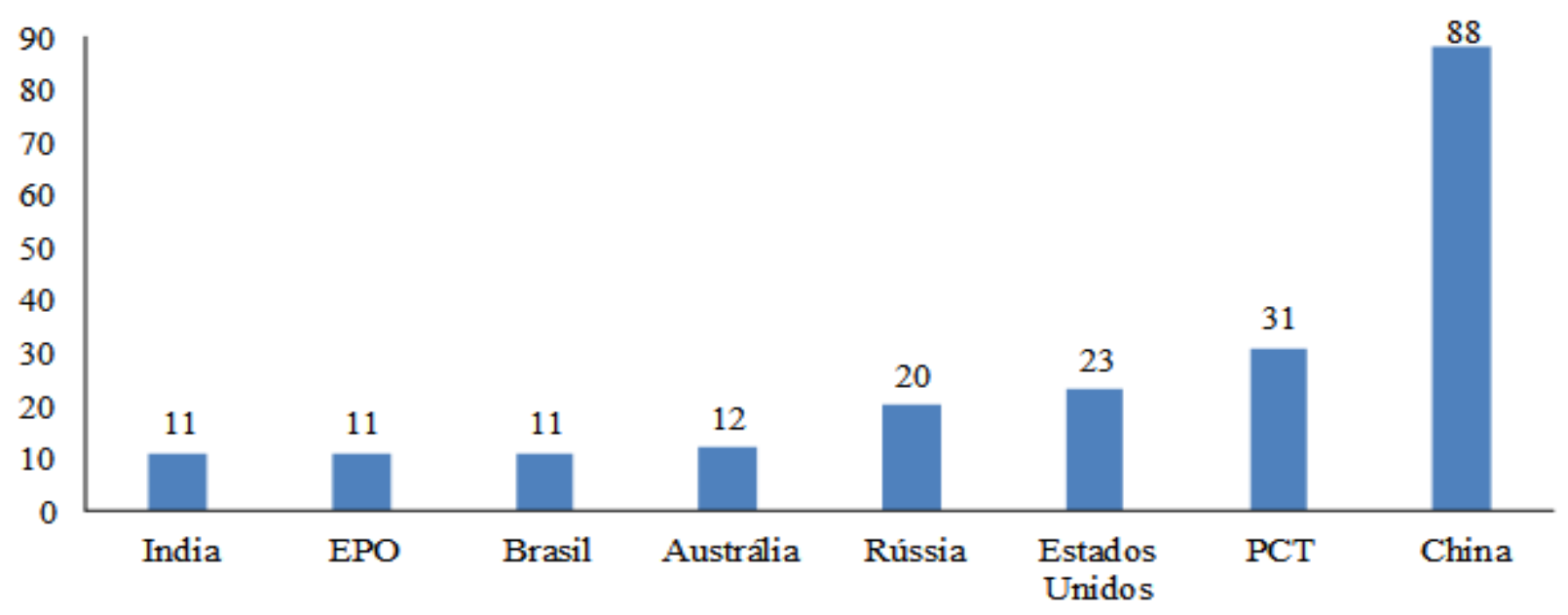

Fonte: Elaborada pelos autores deste artigo (2019)

De acordo com a Figura 2, o Brasil é o sexto maior detentor de pedidos de depósito de patentes sobre "Praziquantel", com 11 patentes, igualando-se com a Índia e a European Patent Office (EPO) - Instituto Europeu de Patentes. A China é a maior detentora, liderando o ranking por países com 88 patentes, seguida do PCT - Tratado de Cooperação de Patentes (31), dos Estados Unidos (23), da Rússia (20) e da Austrália (12). Países como França, Argentina, Canadá, Chile, México, Alemanha, Espanha, Portugal e Japão também aparecem na lista dos pedidos de patentes, porém com quantidades inferiores.

Quanto aos resultados obtidos nas pesquisas realizadas em bancos de dados científicos, de acordo com a Tabela 3, o número total de artigos encontrados nas duas bases utilizadas na pesquisa com todas as palavras-chave foi de 8.256 , sendo bastante superior ao número de patentes depositadas (816) no período de 2008 a 2018, representando apenas 9,87\% da quantidade de artigos publicados. 
Tabela 3 - Número de artigos por palavras-chave em título e resumo recuperadas nos bancos de dados mais consultados no período compreendido de 2008 a 2018

\begin{tabular}{ccc} 
Palavras-chave & Scielo & Scopus \\
Praziquantel & 68 & 4257 \\
Praziquantel and schistosomiasis & 40 & 2283 \\
Praziquantel and anthelmintic & 0 & 1565 \\
Praziquantel and antiparasitics & 0 & 11 \\
Praziquantel and atanine & 0 & 0 \\
Praziquantel and suspension & 0 & 32 \\
TOTAL & 108 & 8148 \\
\hline
\end{tabular}

Fonte: Elaborada pelos autores deste artigo (2019)

A palavra-chave "Praziquantel", assim como na busca por patentes, obteve a maior quantidade de documentos nas bases de dados Scielo e Scopus, obtendo 68 e 4.257 artigos, respectivamente.

Assim como ocorreu com as patentes, a busca por artigos com o descritor "Praziquantel and atanine", de acordo com a Tabela 3, não identificou nenhum trabalho a respeito da associação molecular do fármaco e da molécula em questão, ou seja, o campo para novas pesquisas com o tema está aberto e escasso de informações. É possível o desenvolvimento de trabalhos com a síntese química da molécula, além de testes in vitro e in vivo a fim de testar a toxicidade e a eficácia farmacológica da proposta.

De acordo com a Tabela 3, na plataforma Scielo não houve nenhuma publicação utilizando o termo "Praziquantel and suspension", o que demonstra que no Brasil não há pesquisas sobre a elaboração do PZQ em suspensão, o que se distancia da necessidade por parte do Ministério da Saúde. Assim, de acordo com Furtado (2018), o Praziquantel é produzido no Brasil somente pelo Instituto de Tecnologia e Fármacos (Farmanguinhos/Fiocruz) em comprimidos de 600 mg, distribuídos gratuitamente pelo Sistema Único de Saúde (SUS), embora a Fiocruz apresente uma patente (PI 9804052-9) de 1998 com metodologias de revestimento ou encapsulamento do Praziquantel racêmico para melhorar a rejeição do seu sabor. Ao contrário, na plataforma Scopus já houve 32 resultados usando termo "Praziquantel and suspension", o que retrata estudos científicos por outros países quanto à criação do praziquantel na forma farmacêutica de suspensão.

O maior número de artigos publicados por área de conhecimento para a palavra-chave "Praziquantel" (Figura 3) foi encontrado nas áreas de Medicine (3.188), Immunology and Microbiology (1.334), Pharmacology, Toxicology and Pharmaceutics (518), Biochemistry, Genetics and Molecular Biology (489), Agricultural and Biological Sciences (475), Veterinary (418) e Chemistry (193). O número de 3.188 artigos encontrados em Medicine pode ser justificado porque o praziquantel é um fármaco e possui um grande espectro de ações farmacológicas, além de ser um alvo de interesse para a pesquisa e desenvolvimento de novos medicamentos devido à sua resistência. 
Figura 3 - Áreas de conhecimento baseado na contagem de artigos no banco de dados Scopus com a palavra-chave "Praziquantel" para o período compreendido de 2008 a 2018

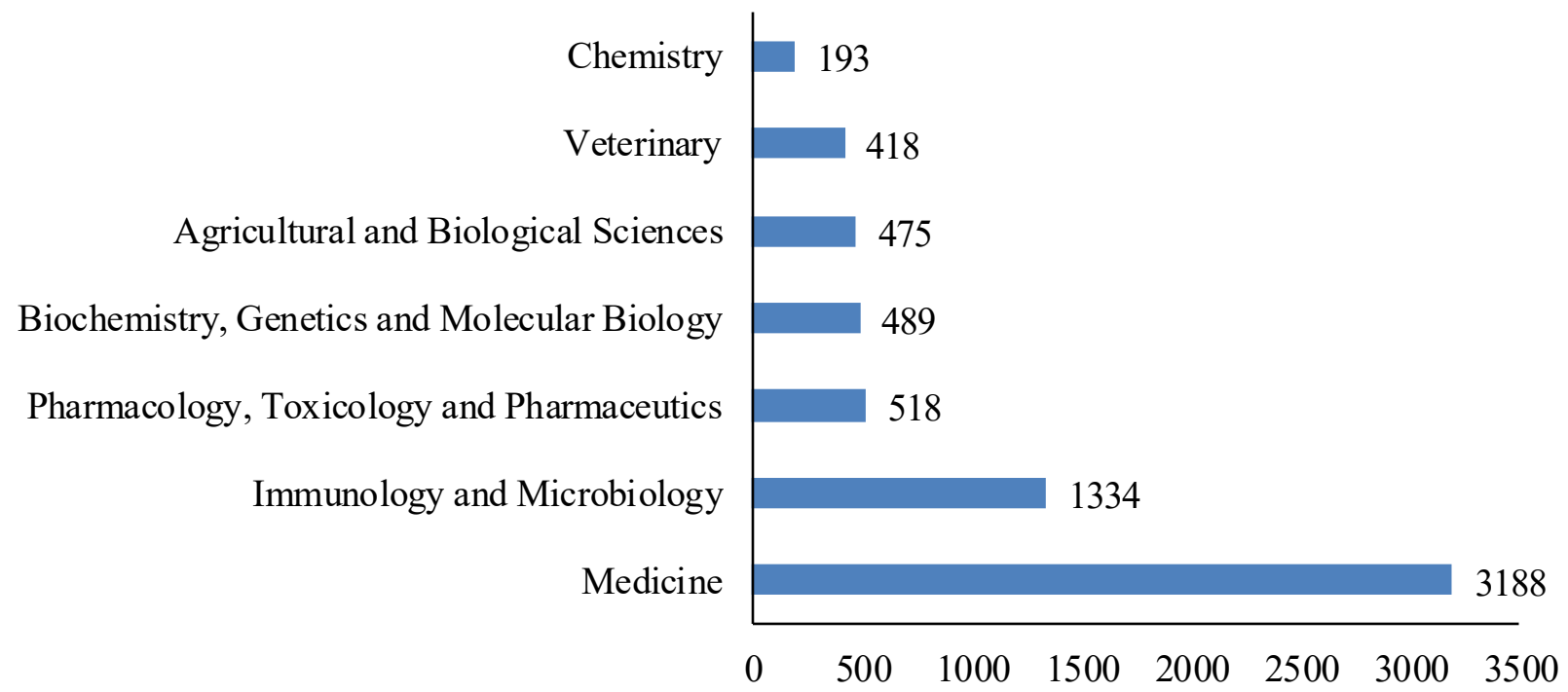

Fonte: Elaborada pelos autores deste artigo (2019)

A Figura 4 quantifica artigos em relação às áreas temáticas propostas pela base de dados Scielo, na qual foram obtidos os seguintes resultados: Ciências da Saúde (55), Ciências Biológicas (32), Ciências Exatas e da Terra (4), Multidisciplinar (3), Ciências Agrárias (1) e Ciências Sociais Aplicadas (1). Com isso, demonstra-se que o PZQ é utilizado não somente para finalidades terapêuticas, mas há interesses em outras áreas.

Figura 4 - Número de artigos publicados por área de conhecimento na base de dados Scielo com a palavra-chave "Praziquantel" no período de 2008 a 2018

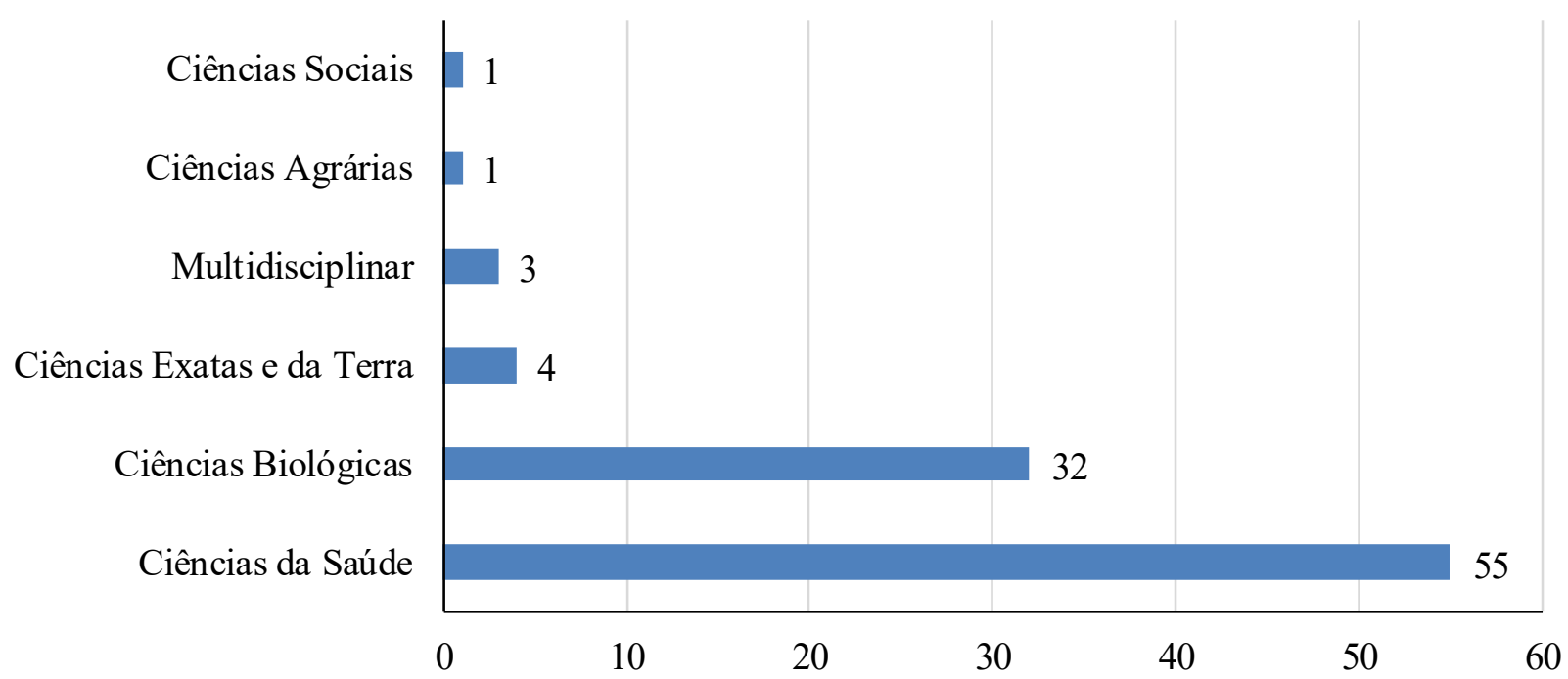

Fonte: Elaborada pelos autores deste artigo (2019)

Somado a isso, a Figura 5 demonstra que o Brasil ocupa o primeiro lugar em quantidade de artigos publicados na plataforma Scielo com 46 documentos, ficando Chile, Cuba, África do Sul e Venezuela em segundo lugar, todos com três artigos cada. 
Figura 5 - Número de documentos de artigos publicados na base Scielo por países com a palavra-chave "Praziquantel" para o período compreendido de 2008 a 2018

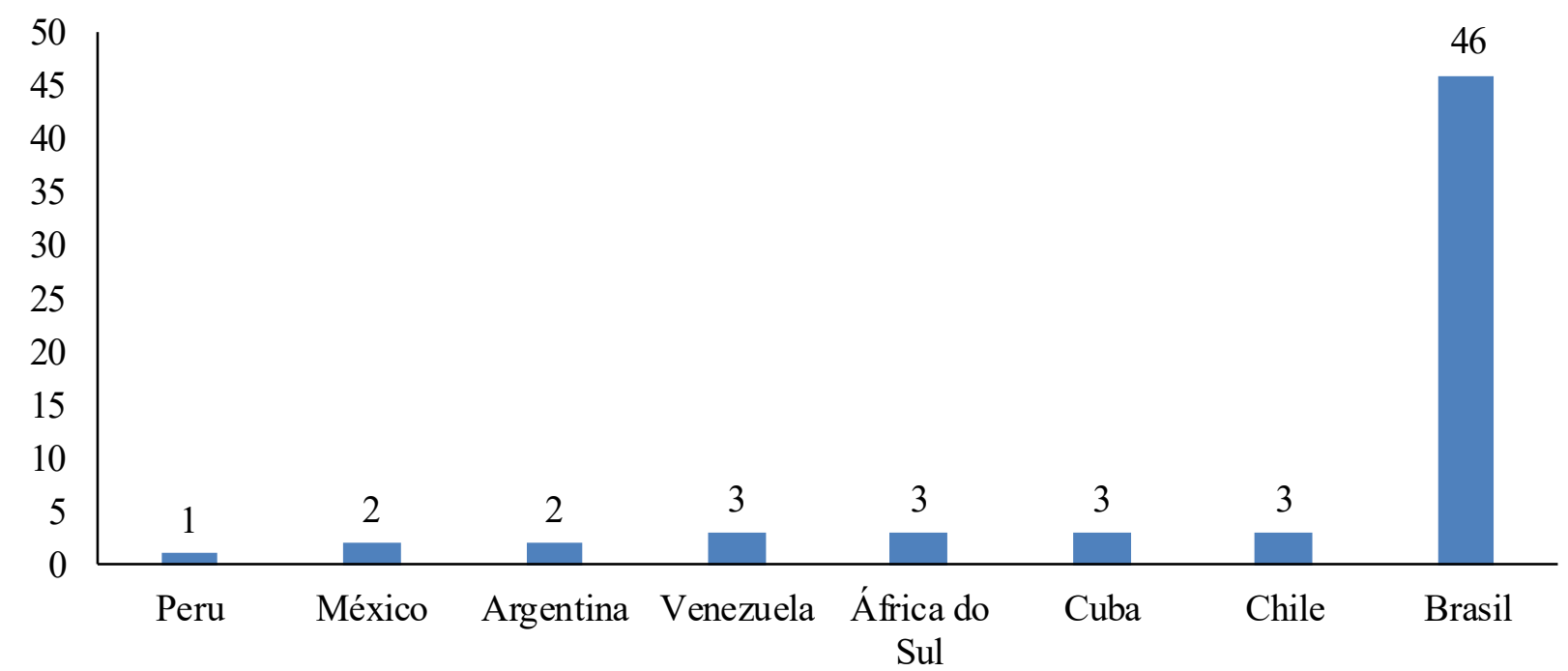

Fonte: Elaborada pelos autores deste artigo (2019)

\section{Considerações Finais}

O número de patentes depositadas relacionadas ao praziquantel é considerado baixo (816) quando esses resultados são comparados com o número de artigos publicados (8.256) sobre essa temática. O Brasil apresentou o maior número de artigos publicados na base de dados Scielo para o período em estudo, entretanto, isso não se reflete aos pedidos de patentes, dos quais países, como Estados Unidos e China, são os maiores detentores.

A base da WIPO é a que detém o maior número de patentes (297) no que se refere às palavras-chave pesquisadas, posteriormente fica a European Patent Office (Espacenet) com 274, a Derwent Innovations Index com 228 patentes e, por fim, a base de dados brasileira, o Instituto Nacional da Propriedade Industrial (INPI) com 17 resultados encontrados.

De um total de 204 patentes para a palavra-chave "Praziquantel", classificadas nas áreas na plataforma Derwent Innovation Index, 99\% encontram-se na área de química e 90\% estão na área de farmacologia.

O Scopus é a base científica com o maior número de documentos relativo à palavra-chave "Praziquantel" e todas as suas combinações. Destes, 3.188 artigos encontram-se na área de Medicina, já na base Scielo, 55 documentos para a área de Ciências da Saúde.

Não há registro de patentes, ou produção científica, relacionado com modificação molecular do praziquantel utilizando a atanina, o que demonstra que este estudo é único e inovador. Além disso, percebe-se que o número de estudos científicos do praziquantel na forma farmacêutica de suspensão foi pequeno quando comparado com a quantidade de patentes e de artigos na combinação "Praziquantel". 


\section{Referências}

ANDREWS, P. Praziquantel: mechanisms of anti-schistosomal activity. Phamacology and Therapeutics, Amsterdam, v. 29, n. 1, p. 129-156, 1985.

ANSEL, H. C.; POPOVICH, N. G.; ALLEN, L. V. Suspensões orais, emulsões, magmas e géis. Farmacotécnica: formas farmacêuticas e sistemas de liberação de fármacos. 6 ed. São Paulo: Editorial Premier, [s.l.], p. 281-315, 2000.

BRASIL. Ministério da Saúde, Portal M.S. Esquistossomose: causas, sintomas, tratamento, diagnóstico e prevenção. Ministério da Saúde, Portal M.S. [2019]. Disponível em: http://portalms. saude.gov.br/saude-de-a-z/esquistossomose. Acesso em: 4 maio 2019.

BRASIL. Ministério da Saúde, Secretaria de Vigilância em Saúde, Departamento de Vigilância epidemiológica. Situação epidemiológica e estratégias de prevenção, controle e eliminação das doenças tropicais negligenciadas no Brasil, 1995 a 2016. Ministério da Saúde, Secretaria de Vigilância em saúde. 4. ed. Brasília: Ministério da Saúde, Brasília, DF, v. 49, n. 49, nov., 2018.

CARVALHO, R. A. et al. Potencialidades farmacológicas da Babosa: um estudo realizado por meio das técnicas de prospecção científica e tecnológica. Cadernos de Prospecção, Salvador, v. 13, n. 1, p. 184-196, março, 2020.

CORDEIRO, Hérica C.; PINHEIRO, José C. Estudo de análogos do praziquantel com atividades contra Schistossoma mansoni através do uso de computador. In: XXVII SEMINÁRIO DE INICIAÇÃO CIENTÍFICA (SEMINIC). Universidade Federal do Pará: Belém, Laboratório de Química Teórica Computacional, 2016. Anais [...]. 2016.

FONSECA, Luís Bastos. Desenvolvimento de nanopartículas poliméricas contendo praziquantel para o tratamento da esquistossomose. 2012. 209f. Tese (Doutorado) - Curso de Engenharia Química, Coope, Universidade Federal do Rio de Janeiro, Rio de Janeiro, 2012.

FURTADO, Daniel Pimenta. Controle e tratamento da esquistossomose no brasil: estudo de caso - praziquantel. Trabalho de Conclusão do Curso (Graduação) - Universidade Federal de Uberlândia, Uberlândia, MG, 2018.

JANNUZZI, Anna Haydée Lanzillotti; SOUZA, Cristina Gomes de. Patentes de invenção e artigos científicos: especificidades e similitudes. Revista Brasileira de Pós-Graduação, Brasília, DF, v. 5, n. 9, p. 103-125, dezembro de 2008.

KANDIL, O. The pharmaceutical industry in the Arab Word challenger, controversies and future Outlook. Drug Discovery Today, [s.l.], v. 9, n. 13, p. 543-545, 2004.

LEOPOLD, G. et al. Clinical pharmacology in normal volunteers of praziquantel, a new drug against shistosomes and cestodes. An example of a complex study covering both tolerance and pharmaco kinetica. European Journal of Clinical Pharmacology, Berlim, v. 14, n. 4, p. 281-291, 1978.

MERCK INDEX. 13. Índice Merck de produtos químicos e droga, Livro. 13 ed. New Jersey: Merck E. Corporationed. New Jersey: Merck E. Corporation, [s.l.], p. 1.174, 2001.

NASCIMENTO, Jaqueline S. et al. Estudo prospectivo relativo à atividade antifúngica de Schinus terebinthifolius (aroeira) no período de 1990 a 2016. Cadernos de Prospecção, Salvador, v. 10, n. 4, p. 839-850, out.-dez. 2017. 
PEREIRA, S. A. et al. Prospecção científica e tecnológica do gênero Jatropha (Euphorbiaceae).

Cadernos de Prospecção, Salvador, v. 8, n. 2, p. 355-364, abr.-jun. 2015.

SANTOS, C. S. et al. Representações sociais de profissionais de saúde sobre doenças negligenciadas. Escola Anna Nery, Rio de janeiro, v. 21, n. 1, p. 1-9, fev. 2017.

SILVA, Andreza de Almeida e. Preparação e caracterização de granulados contendo dispersão sólida de praziquantel obtidos por fusão/solidificação em leite fluidizado. 2008. $158 \mathrm{f}$. Dissertação (Mestrado) - Curso de Farmácia, Universidade de São Paulo, Ribeirão Preto, 2008.

SILVA, E. L.; NICOLETTI, M. A. Controle e tratamento das doenças negligenciadas: visão da situação atual. Rev. Saúde, [s.l.], v. 7, n. 3/4, p. 65-81, 2013.

SILVA, Vinícius B. R. et al. Medicinal chemistry of antischistosomal drugs: Praziquantel and oxamniquine. Bioorganic \& Medicinal Chemistry, [s.l.], v. 25, p. 3.259-3.277, 2017.

SOCIEDADE BRASILEIRA DE INFECTOLOGIA. Esquistossomose. São Paulo: SBI, 2018.

Disponível em: https://www.infectologia.org.br/pg/981/esquistossomose. Acesso em: 4 maio 2019.

WOELFLE, M. et al. Resolution of Praziquantel. PLOS Neglected Tropical Diseases, [s.l.], v. 5, n. 9, p. 1-7, 2011.

\section{Sobre os Autores}

\section{Pedro da Silva Gerônimo Neto}

E-mail: pedronetogeronimo@gmail.com

Graduando do curso de Farmácia da Universidade Federal do Maranhão.

Endereço profissional: Avenida dos Portugueses, n. 1.966, Coordenação do Curso de Farmácia - CCBS, Campus do Bacanga, São Luis, MA. CEP: 65080-805.

\section{João Marcelo Saraiva Ferreira}

E-mail: marcelosaraiva1998@gmail.com

Graduando do curso de Farmácia da Universidade Federal do Maranhão.

Endereço profissional: Avenida dos Portugueses, n. 1.966, Coordenação do Curso de Farmácia - CCBS, Campus do Bacanga, São Luís, MA. CEP: 65080-805.

\section{Pablo de Matos Monteiro}

E-mail: pablomonteiro50@gmail.com

Graduando curso de Farmácia da Universidade Federal do Maranhão.

Endereço profissional: Avenida dos Portugueses, n. 1.966, Coordenação do Curso de Farmácia - CCBS, Campus do Bacanga, São Luís, MA. CEP: 65080-805.

\section{Maria da Glória Almeida Bandeira}

E-mail:mgban10@yahoo.com.br

Doutora em Biologia de Água Doce e Pesca Interior.

Endereço profissional: Avenida dos Portugueses, n. 1.966, Departamento de Tecnologia Química - CCET, Campus do Bacanga, São Luís, MA. CEP: 65080-805. 


\section{Jaqueline Silva Nascimento}

E-mail: jackdapi@gmail.com

Graduada em Turismo - Turismólogo.

Endereço profissional: Avenida Colares Moreira, 477, Pró-Reitoria de Pesquisa, Pós-graduação e Inovação PRPGI, Reitoria do IFMA, Renascença, São Luís, MA. CEP: 65075-441. 